\title{
9-乙酰氧基乙基-6-烷氨基-2-烷硫基嘌呤的合成及抗血小板凝集活性评估
}

\author{
何琦文杜洪光* \\ (北京化工大学理学院 北京 100029)
}

\begin{abstract}
摘要 以 2-氨基-6-氯嘌呤(1)为原料, 与 2-溴乙基乙酸酯反应得到 9-乙酰氧基乙基-2-氨基-6-氯嘌呤(2); 2 经重氮-烷硫 化得到 9-乙酰氧基乙基-2-烷硫基-6-氯嘌呤(3); 3 经胺解反应得到 14 个未见报道的 9-乙酰氧基乙基-6-烷氨基-2-烷硫基 嘌呤化合物 4. 这些化合物的结构经 IR, ${ }^{1} \mathrm{H} N M R,{ }^{13} \mathrm{C} N M R$ 及 HRMS 得到表征, 同时进行了抗血小板凝集活性测试. 关键词 6-烷胺基-2-烷硫基嘌呤; 合成; 结构表征; 抗血小板凝集
\end{abstract}

\section{Synthesis of 9-Acetoxyethyl-6-alkylamino-2-alkylthio Purines and Their Anti-platelet Aggregation Activity}

\author{
He, Qiwen Du, Hongguang* \\ (College of Science, Beijing University of Chemical Technology, Beijing 100029)
}

\begin{abstract}
Amino-6-chloropurine (1) as the starting material was treated with 2-bromoethyl acetate to yield 9-acetoxyethyl-2-amino-6-chloropurine (2). Compound 2 was diazotized and reacted with disulfides to afford 9-acetoxyethyl-2-alkythio-6-chloropurine (3). 9-Acetoxyethyl-6-alkylamino-2-alkylthio purines (4) were acquired by aminolysis reaction of compounds 3. Their structures were determined by IR, ${ }^{1} \mathrm{H}$ NMR, ${ }^{13} \mathrm{C}$ NMR and HRMS techniques. Meanwhile, the anti-platelet aggregation rates of compounds 4 were measured.
\end{abstract}

Keywords 6-alkylamino-2-alkylthio purine; synthesis; characterization; anti-platelet aggregation

嘌呤类化合物在自然界中广泛存在，它们不仅在生 命体的遗传和代谢等多种生命活动中起着至关重要的 作用 ${ }^{[1]}$, 同时在抗病毒、抗肿瘤和抗血栓等方面也具有 良好的生物活性 ${ }^{[2 \sim 5]}$, 因此对于嘌呤化合物的研究一直 是人们关注的焦点. 经研究表明, 在嘌呤环上引入某些 基团, 可以得到具有某些生物活性的医药中间体和重要 药物 ${ }^{[6,7]}$. 如已用于临床的抗疮疹病毒药物阿昔洛韦 ${ }^{[8]}$, 其替代药物更昔洛韦和喷昔洛韦 ${ }^{[9]}$ 以及抗血栓药物坎格 雷洛和替卡格雷洛等 ${ }^{[10,11]}$.

早在 1973 年, Kikugawa 研究小组 ${ }^{[12]}$ 就对 2-烷硫基 嘌呤核苷化合物进行了抗血小板凝集活性的研究, 结果 表明 2-烷硫基腺苷类化合物可以很好的抑制 ADP 诱导 的血小板凝聚. 之后 Ingall 等 ${ }^{[13]}$ 通过研究发现, 在嘌呤 环的 C-6 位上引入烷氨基也可对抗血小板凝集活性的提 高有一定作用.

2003 年 Kim 等 ${ }^{[14]}$ 报道了以 2 -氨基-6-氯嘌呤为原料 经 9 位光延反应, 2 位重氮化反应和 6 位胺化反应得到多 取代的嘌呤化合物, 并研究了其对 $\mathrm{P} 2 \mathrm{Y}_{1}$ 受体的抑制作
用. 2006 年 Rehse 等 ${ }^{[15]}$ 制备了一系列的 2-酰胺基-6-取代 氨基-9-芐基嘌呤类化合物, 并对这些化合物进行了抑 制血小板凝聚活性的评价，篮选出了一种对抑制 ADP 诱导的血小板凝集有显著效果的的化合物, 其 $\mathrm{IC}_{50}=$ $0.00045 \mu \mathrm{mol} / \mathrm{L}$.

在 2011 年, 我们研究小组 ${ }^{[16,17]}$ 分别报道了以鸟苷 为原料经羟基保护、重氮化、水解、 $O$-烷基化和胺解反 应合成 2-烷氧基腺苷化合物和以腺嘌呤为原料合成 2烷硫基腺嘌呤化合物，同时测试了部分化合物的抗血小 板凝集活性，得到了一个血小板最大凝集率仅为 $6.15 \%$ 的 6-烷氨基腺苷化合物 ${ }^{[16]}$.

然而到目前为止，对于 6-烷氨基-2-烷硫基嘌呤化 合物的合成及生物活性的研究仍相对较少. 本文以 2-氨 基-6-氯嘌呤(1)为原料设计合成了 14 种未见报道的 9-乙 酰氧基乙基-6-烷氨基-2-烷硫基嘌呤化合物，并通过 IR, ${ }^{1} \mathrm{H}$ NMR, ${ }^{13} \mathrm{C}$ NMR 及 HRMS 等手段对其结构进行了表 征, 且对这 14 种化合物的抗血小板凝集活性进行了测 试. 合成路线如 Scheme 1 所示.

*E-mail: dhg@mail.buct.edu.cn

Received March 9, 2012; revised April 22, 2012; published online May 22, 2012. 


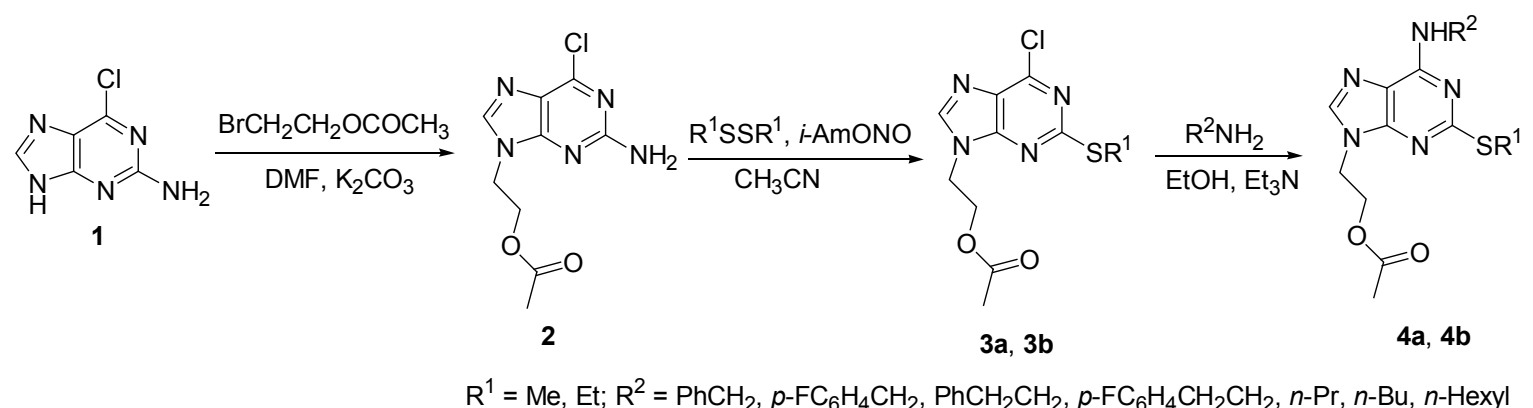

Scheme 1

\section{1 实验部分}

\section{1 仪器与试剂}

XT-4A 型显微熔点测定仪, 上海精密科学仪器有限 公司(温度计未校正); 岛津-4000 型傅里叶变换红外光 谱仪, 日本岛津公司; Varian $300 \mathrm{MHz}$ 核磁共振仪, 美 国 Varian 公司; Bruker $400 \mathrm{MHz}$ 核磁共振仪, 德国 Bruker 公司; Agilent TOF-6210 型高分辨质谱仪, 美国 Agilent 公司. 2-氨基-6-氯嘌呤, 济南明金制药有限公司; 青岛海洋化工厂硅胶(200 300 目); 溴乙醇、亚硝酸异 戊酯、二甲基二硫醚、二乙基二硫醚、芐胺、三乙胺、 无水碳酸钾等为化学纯；2-溴乙基乙酸酯按文献 [18]方 法制备.

\section{2 实验方法}

\subsubsection{9-乙酰氧基乙基-2-氨基-6-氯嘌呤(2)的合成}

将 $10.0 \mathrm{~g}(59.0 \mathrm{mmol})$ 2-氨基-6-氯嘌呤、 $10.0 \mathrm{~g}$ (72.4 $\mathrm{mmol})$ 无水碳酸钾和 $14.8 \mathrm{~g}(88.6 \mathrm{mmol}) 2$-溴乙基乙酸酯 加入到 $150 \mathrm{~mL}$ DMF 中, 室温搅拌反应 $20 \mathrm{~h}$. 将过量的 碳酸钾滤出, 减压蒸馏除去 DMF, 粗产物再用水洗 3 次, 干燥后加入 $300 \mathrm{~mL}$ 无水乙醇重结晶, 得到 $10.5 \mathrm{~g}$ 白色 固体 2, 产率 $65.6 \%$. m.p. $179 \sim 181{ }^{\circ} \mathrm{C} ;{ }^{1} \mathrm{H}$ NMR (300 MHz, DMSO- $\left.d_{6}\right) \delta: 8.15$ (s, $\left.1 \mathrm{H}, \mathrm{H}-8\right), 6.95$ (bs, $2 \mathrm{H}, \mathrm{NH}_{2}$ ), $4.40 \sim 4.28\left(\mathrm{~m}, 4 \mathrm{H}, \mathrm{NCH}_{2} \mathrm{CH}_{2} \mathrm{O}\right), 1.97\left(\mathrm{~s}, 3 \mathrm{H}, \mathrm{COCH}_{3}\right)$; ${ }^{13} \mathrm{C}$ NMR (100 MHz, DMSO- $\left.d_{6}\right) \delta: 170.11,159.78$, 154.23, 149.33, 143.29, 123.20, 61.66, 42.25, 20.51.

\subsubsection{9-乙酰氧基乙基-2-烷硫基-6-氯嘌呤(3)的合成}

将 $0.5 \mathrm{~g}(2.0 \mathrm{mmol})$ 化合物 2 和 $1.2 \mathrm{~g}(11.8 \mathrm{mmol})$ 二 甲基二硫醚加入到 $20 \mathrm{~mL}$ 无水乙腈中, 在氮气保护下室 温摚拌 $10 \mathrm{~min}$, 之后加入 $2.4 \mathrm{~g}(19.6 \mathrm{mmol})$ 亚硝酸异戊 酷继续摚拌 $5 \mathrm{~min}$, 然后置于 $65{ }^{\circ} \mathrm{C}$ 的油浴中反应 $3 \mathrm{~h}$. 减压蒸除溶剂, 经柱色谱分离 $[V($ 乙酸乙酯) $: V($ 石油 醚) $=1: 2 \sim 2: 3$ )得到化合物 3a. 参照上述方法用二乙 基二硫醚代替二甲基二硫醚即可得到化合物 $\mathbf{3 b}$.

9-乙酰氧基乙基-2-甲硫基-6-氯嘌呤 (3a): 白色固 体, 产率 64.2\%. m.p. $110 \sim 112{ }^{\circ} \mathrm{C} ;{ }^{1} \mathrm{H}$ NMR (400 MHz,
$\left.\mathrm{CDCl}_{3}\right) \delta: 7.99(\mathrm{~s}, \quad 1 \mathrm{H}, \mathrm{H}-8), 4.52 \sim 4.44(\mathrm{~m}, 4 \mathrm{H}$, $\mathrm{NCH}_{2} \mathrm{CH}_{2} \mathrm{O}$ ), 2.64 (s, 3H, $\left.\mathrm{SCH}_{3}\right), 2.05$ (s, 3H, $\mathrm{COCH}_{3}$ ); ${ }^{13} \mathrm{C}$ NMR (100 MHz, $\left.\mathrm{CDCl}_{3}\right) \delta: 170.33,166.60,152.63$, $150.85,143.92,128.41,61.87,43.02,20.70,14.75$.

9-乙酰氧基乙基-2-乙硫基-6-氯嘌呤 $(3 \mathbf{b})$ : 淡黄色固 体, 产率 59.8\%. m.p. $75 \sim 77{ }^{\circ} \mathrm{C}$; ${ }^{1} \mathrm{H}$ NMR $(400 \mathrm{MHz}$, $\left.\mathrm{CDCl}_{3}\right) \delta: 7.95(\mathrm{~s}, 1 \mathrm{H}, \mathrm{H}-8), 4.43 \sim 4.40(\mathrm{~m}, 4 \mathrm{H}$, $\mathrm{NCH}_{2} \mathrm{CH}_{2} \mathrm{O}$ ), 3.18 (q, $J=7.3 \mathrm{~Hz}, 2 \mathrm{H}, \mathrm{SCH}_{2}$ ), 2.00 (s, $3 \mathrm{H}$, $\left.\mathrm{COCH}_{3}\right), 1.40\left(\mathrm{t}, J=7.3 \mathrm{~Hz}, 3 \mathrm{H}, \mathrm{SCH}_{2} \mathrm{CH}_{3}\right) ;{ }^{13} \mathrm{C} \mathrm{NMR}$ $\left(100 \mathrm{MHz}, \mathrm{CDCl}_{3}\right) \delta: 170.33,166.23,152.64,150.92$, $143.89,128.47,61.87,43.02,25.97,20.70,14.27$.

1.2.39-乙酰氧基乙基-6-烷氨基-2-烷硫基嘌呤(4)的 合成

在装有电磁搅拌、温度计和回流冷凝管的三口瓶中, 加入 $300.0 \mathrm{mg}(1.1 \mathrm{mmol})$ 化合物 $\mathbf{3 a}, 170.0 \mathrm{mg}(1.6 \mathrm{mmol})$ 芐胺, $160.0 \mathrm{mg}(1.6 \mathrm{mmol})$ 三乙胺和 $15 \mathrm{~mL}$ 乙醇, 回流 $1.5 \mathrm{~h}$. 减压除去溶剂后, 加入 $50 \mathrm{~mL}$ 水并调节 $\mathrm{pH}$ 至中 性, 用 $15 \mathrm{~mL}$ 乙酸乙酯萃取 3 次后, 经柱色谱分离 $[V($ 乙 酸乙酯) $: V$ (二氯甲烷 $)=1.0: 1.5$ ]得到化合物 $\mathbf{4 a}_{1}$, 参照 上述方法分别用对氟苄胺、苯乙胺、对氟苯乙胺、丙胺、 正丁胺、正己胺等代替苠胺制得化合物 $\mathbf{4 a}_{2} \sim \mathbf{4 a}_{7}$. 同理, 用 $3 b$ 分别与上述胺反应可得到化合物 $\mathbf{4} b_{1} \sim 4 b_{7}$.

9-乙酰氧基乙基-6-芐氨基-2-甲硫基嘌呤 $\left(\mathbf{4 a}_{\mathbf{1}}\right)$ : 白 色固体, m.p. $171 \sim 173{ }^{\circ} \mathrm{C}$, 产率 $91.2 \%$. ${ }^{1} \mathrm{H}$ NMR (400 $\left.\mathrm{MHz}, \mathrm{CDCl}_{3}\right) \delta: 7.42(\mathrm{~s}, 1 \mathrm{H}, \mathrm{H}-8), 7.36 \sim 7.25(\mathrm{~m}, 5 \mathrm{H}$, $\mathrm{ArH}), 6.72$ (bs, $1 \mathrm{H}, \mathrm{NH}$ ), 4.84 (bs, $2 \mathrm{H}, \mathrm{NHCH}_{2}$ ), $4.40 \sim$ $4.32\left(\mathrm{~m}, 4 \mathrm{H}, \mathrm{NCH}_{2} \mathrm{CH}_{2} \mathrm{O}\right), 2.55$ (s, $\left.3 \mathrm{H}, \mathrm{SCH}_{3}\right), 2.01(\mathrm{~s}, 3 \mathrm{H}$, $\left.\mathrm{COCH}_{3}\right) ;{ }^{13} \mathrm{C}$ NMR $\left(100 \mathrm{MHz}, \mathrm{CDCl}_{3}\right) \delta: 170.49,165.87$, 153.96, 149.34, 138.93, 138.84, 128.58, 127.77, 127.37, $117.15,62.37,44.43,42.38,20.77,14.60$; IR (KBr) $v$ : 3254, 2921, 1724, 1617, 1577, 1337, 1299, 1227, 771 $\mathrm{cm}^{-1}$; HRMS (ESI) calcd for $\mathrm{C}_{17} \mathrm{H}_{20} \mathrm{~N}_{5} \mathrm{O}_{2} \mathrm{~S}[\mathrm{M}+\mathrm{H}]^{+}$ 358.1332, found 358.1333 .

9-乙酰氧基乙基-6-对氟芐氨基-2-甲硫基嘌呤 $\left(\mathbf{4} \mathbf{a}_{2}\right)$ : 白色固体, m.p. 132 134 ${ }^{\circ} \mathrm{C}$, 产率 $86.5 \% .{ }^{1} \mathrm{H}$ NMR (400 
$\left.\mathrm{MHz}, \mathrm{CDCl}_{3}\right) \delta: 7.51(\mathrm{~s}, 1 \mathrm{H}, \mathrm{H}-8), 7.32 \sim 7.27(\mathrm{~m}, 2 \mathrm{H}$, ArH), $7.00 \sim 6.95(\mathrm{~m}, 2 \mathrm{H}, \mathrm{ArH}), 6.61(\mathrm{~s}, 1 \mathrm{H}, \mathrm{NH}), 4.80$ $\left(\mathrm{bs}, 2 \mathrm{H}, \mathrm{NHCH}_{2}\right), 4.42 \sim 4.35\left(\mathrm{~m}, 4 \mathrm{H}, \mathrm{NCH}_{2} \mathrm{CH}_{2} \mathrm{O}\right), 2.54$ (s, 3H, $\left.\mathrm{SCH}_{3}\right), 2.02$ (s, 3H, $\left.\mathrm{COCH}_{3}\right) ;{ }^{13} \mathrm{C} \mathrm{NMR}(100 \mathrm{MHz}$, $\left.\mathrm{CDCl}_{3}\right) \delta: 170.47,165.91,160.88,153.85,150.45,138.97$, $134.58,129.43,129.35,117.13,115.47,115.26,62.35$, 43.74, 42.40, 20.74, 14.55; IR (KBr) v: 3258, 2924, 1726, 1617, 1579, 1338, 1299, 1221, $770 \mathrm{~cm}^{-1}$; HRMS (ESI) calcd for $\mathrm{C}_{17} \mathrm{H}_{19} \mathrm{FN}_{5} \mathrm{O}_{2} \mathrm{~S}[\mathrm{M}+\mathrm{H}]^{+}$376.1238, found 376.1237 .

9-乙酰氧基乙基-6-苯乙氨基-2-甲硫基嘌呤 $\left(\mathbf{4} \mathbf{a}_{3}\right)$ : 白色固体, m.p. 124 $126{ }^{\circ} \mathrm{C}$, 产率 79.8\%. ${ }^{1} \mathrm{H}$ NMR (400 $\left.\mathrm{MHz}, \mathrm{CDCl}_{3}\right) \delta: 7.54(\mathrm{~s}, 1 \mathrm{H}, \mathrm{H}-8), 7.31 \sim 7.19(\mathrm{~m}, 5 \mathrm{H}$, ArH), 6.05 (bs, 1H, NH), $4.43 \sim 4.35$ (m, 4H, $\mathrm{NCH}_{2} \mathrm{CH}_{2} \mathrm{O}$ ), 3.89 (bs, 2H, $\mathrm{NHCH}_{2}$ ), 2.97 (t, $J=7.1 \mathrm{~Hz}$, $\left.2 \mathrm{H}, \mathrm{ArCH}_{2}\right), 2.59\left(\mathrm{~s}, 3 \mathrm{H}, \mathrm{SCH}_{3}\right), 2.03\left(\mathrm{~s}, 3 \mathrm{H}, \mathrm{COCH}_{3}\right) ;{ }^{13} \mathrm{C}$ NMR $\left(100 \mathrm{MHz}, \mathrm{CDCl}_{3}\right) \delta: 170.50,165.79,154.08$, 138.78, 128.84, 128.62, 126.46, 117.24, 62.41, 42.37, 41.99, 35.99, 20.78, 14.60; IR (KBr) v: 3269, 2925, 1743, 1614, 1581, 1332, 1301, 1228, $772 \mathrm{~cm}^{-1}$; HRMS (ESI) calcd for $\mathrm{C}_{18} \mathrm{H}_{22} \mathrm{~N}_{5} \mathrm{O}_{2} \mathrm{~S}[\mathrm{M}+\mathrm{H}]^{+}$372.1489, found 372.1492 .

9-乙酰氧基乙基-6-对氟苯乙氨基-2-甲硫基嘌呤 $\left(4 \mathbf{a}_{4}\right)$ : 白色固体, m.p. $112 \sim 114{ }^{\circ} \mathrm{C}$, 产率 $87.5 \%$. ${ }^{1} \mathrm{H}$ NMR (400 MHz, $\left.\mathrm{CDCl}_{3}\right) \delta: 7.59(\mathrm{~s}, 1 \mathrm{H}, \mathrm{H}-8), 7.21 \sim 7.16$ $(\mathrm{m}, 2 \mathrm{H}, \mathrm{ArH}), 7.01 \sim 6.94(\mathrm{~m}, 2 \mathrm{H}, \mathrm{ArH}), 5.89$ (bs, 1H, $\mathrm{NH}), 4.43 \sim 4.36\left(\mathrm{~m}, 4 \mathrm{H}, \mathrm{NCH}_{2} \mathrm{CH}_{2} \mathrm{O}\right), 3.87$ (bs, $2 \mathrm{H}$, $\left.\mathrm{NHCH}_{2}\right), 2.95$ (t, J=7.1 Hz, 2H, $\left.\mathrm{ArCH}_{2}\right), 2.59$ (s, 3H, $\left.\mathrm{SCH}_{3}\right), 2.03$ (s, 3H, $\left.\mathrm{COCH}_{3}\right) ;{ }^{13} \mathrm{C} \mathrm{NMR}\left(100 \mathrm{MHz}, \mathrm{CDCl}_{3}\right)$ $\delta: 170.49,165.83,160.43,154.05,138.83,134.64,130.27$, 130.19, 117.23, 115.50, 115.29, 62.39, 42.38, 42.04, 35.17, 20.77, 14.57; IR (KBr) v: 3268, 2925, 1743, 1612, 1582, 1327, 1302, 1220, $770 \mathrm{~cm}^{-1}$; HRMS (ESI) calcd for $\mathrm{C}_{18} \mathrm{H}_{21} \mathrm{FN}_{5} \mathrm{O}_{2} \mathrm{~S}[\mathrm{M}+\mathrm{H}]^{+}$390.1395, found 390.1399.

9-乙酰氧基乙基-6-丙氨基-2-甲硫基嘌呤 $\left(4 \mathbf{a}_{5}\right)$ ：白 色固体, m.p. 166 168 ${ }^{\circ} \mathrm{C}$, 产率 $88.7 \%$. ${ }^{1} \mathrm{H}$ NMR (400 $\left.\mathrm{MHz}, \mathrm{CDCl}_{3}\right) \delta: 7.66$ (s, 1H, H-8), 5.91 (bs, 1H, NH), $4.42 \sim 4.34\left(\mathrm{~m}, 4 \mathrm{H}, \mathrm{NCH}_{2} \mathrm{CH}_{2} \mathrm{O}\right), 3.57$ (bs, $2 \mathrm{H}, \mathrm{NHCH}_{2}$ ), $2.54\left(\mathrm{~s}, 3 \mathrm{H}, \mathrm{SCH}_{3}\right), 2.01\left(\mathrm{~s}, 3 \mathrm{H}, \mathrm{COCH}_{3}\right), 1.67$ (sextet, $J=$ $7.2 \mathrm{~Hz}, 2 \mathrm{H}, \mathrm{NHCH}_{2} \mathrm{CH}_{2}$ ), 0.97 (t, $J=7.2 \mathrm{~Hz}, 3 \mathrm{H}$, $\left.\mathrm{NH}\left(\mathrm{CH}_{2}\right)_{2} \mathrm{CH}_{3}\right) ;{ }^{13} \mathrm{C}$ NMR $\left(100 \mathrm{MHz}, \mathrm{CDCl}_{3}\right) \delta: 170.46$, $165.97,154.00,150.04,138.66,116.76,62.33,42.45$, 39.60, 22.97, 20.75, 14.47, 11.42; IR (KBr) v: 3263, 2959, 2923, 1730, 1621,1578, 1339, 1298, $1238 \mathrm{~cm}^{-1}$; HRMS (ESI) calcd for $\mathrm{C}_{13} \mathrm{H}_{20} \mathrm{~N}_{5} \mathrm{O}_{2} \mathrm{~S}[\mathrm{M}+\mathrm{H}]^{+}$310.1332, found
310.1332 .

9-乙酰氧基乙基-6-正丁氨基-2-甲硫基嘌呤 $\left(4 \mathbf{a}_{6}\right)$ : 白色固体, m.p. $134 \sim 136{ }^{\circ} \mathrm{C}$, 产率 $84.5 \%$. ${ }^{1} \mathrm{H}$ NMR (400 $\left.\mathrm{MHz}, \mathrm{CDCl}_{3}\right) \delta: 7.60$ (s, 1H, H-8), 5.79 (bs, 1H, NH), $4.40 \sim 4.34\left(\mathrm{~m}, 4 \mathrm{H}, \mathrm{NCH}_{2} \mathrm{CH}_{2} \mathrm{O}\right), 3.61$ (bs, $\left.2 \mathrm{H}, \mathrm{NHCH}_{2}\right)$, $2.54\left(\mathrm{~s}, 3 \mathrm{H}, \mathrm{SCH}_{3}\right), 2.00\left(\mathrm{~s}, 3 \mathrm{H}, \mathrm{COCH}_{3}\right), 1.61$ (quintet, $J=7.3 \mathrm{~Hz}, 2 \mathrm{H}, \mathrm{NHCH}_{2} \mathrm{CH}_{2}$ ), 1.39 (sextet, $J=7.4 \mathrm{~Hz}, 2 \mathrm{H}$, $\left.\mathrm{NH}\left(\mathrm{CH}_{2}\right)_{2} \mathrm{CH}_{2}\right), 0.91$ (t, $\left.J=7.2 \mathrm{~Hz}, 3 \mathrm{H}, \mathrm{NH}\left(\mathrm{CH}_{2}\right)_{3} \mathrm{CH}_{3}\right)$; ${ }^{13} \mathrm{C}$ NMR $\left(100 \mathrm{MHz}, \mathrm{CDCl}_{3}\right) \delta: 170.47,165.77,154.25$, 138.62, 117.16, 62.40, 42.34, 40.26, 31.83, 20.75, 20.04, 14.52, 13.81; IR (KBr) v: 3274, 2962, 2927, 1731, 1618, $1578,1338,1297 \mathrm{~cm}^{-1}$; HRMS (ESI) calcd for $\mathrm{C}_{14} \mathrm{H}_{22} \mathrm{~N}_{5^{-}}$ $\mathrm{O}_{2} \mathrm{~S}[\mathrm{M}+\mathrm{H}]^{+}$324.1489, found 324.1491.

9-乙酰氧基乙基-6-正己氨基-2-甲硫基嘌呤 $\left(\mathbf{4} \mathbf{a}_{7}\right)$ : 白色固体, m.p. $125 \sim 127{ }^{\circ} \mathrm{C}$, 产率 78.7\%. ${ }^{1} \mathrm{H}$ NMR (400 $\left.\mathrm{MHz} \mathrm{CDCl}_{3}\right) \delta: 7.60$ (s, 1H, H-8), 5.76 (bs, 1H, NH), $4.41 \sim 4.34\left(\mathrm{~m}, 4 \mathrm{H}, \mathrm{NCH}_{2} \mathrm{CH}_{2} \mathrm{O}\right), 3.60$ (bs, $2 \mathrm{H}, \mathrm{NHCH}_{2}$ ), $2.55\left(\mathrm{~s}, 3 \mathrm{H}, \mathrm{SCH}_{3}\right), 2.01\left(\mathrm{~s}, 3 \mathrm{H}, \mathrm{COCH}_{3}\right), 1.62$ (quintet, $\left.J=7.3 \mathrm{~Hz}, 2 \mathrm{H}, \mathrm{NHCH}_{2} \mathrm{CH}_{2}\right), 1.41 \sim 1.33(\mathrm{~m}, 2 \mathrm{H}$, $\left.\mathrm{NH}\left(\mathrm{CH}_{2}\right)_{2} \mathrm{CH}_{2}\right), 1.32 \sim 1.25\left(\mathrm{~m}, 4 \mathrm{H}, \mathrm{NH}\left(\mathrm{CH}_{2}\right)_{3} \mathrm{CH}_{2} \mathrm{CH}_{2}\right)$, $0.86\left(\mathrm{t}, J=6.9 \mathrm{~Hz}, 3 \mathrm{H}, \mathrm{NH}\left(\mathrm{CH}_{2}\right)_{5} \mathrm{CH}_{3}\right) ;{ }^{13} \mathrm{C}$ NMR $(100$ $\left.\mathrm{MHz}, \mathrm{CDCl}_{3}\right) \delta: 170.48,165.80,154.26,138.61,117.19$, $62.41,42.35,40.57,31.55,29.70,26.60,22.59,20.76$, 14.52, 14.03; IR (KBr) v: 3268, 2960, 2923, 1732, 1621, 1580, 1339, 1297, $1235 \mathrm{~cm}^{-1}$; HRMS (ESI) calcd for $\mathrm{C}_{16} \mathrm{H}_{26} \mathrm{~N}_{5} \mathrm{O}_{2} \mathrm{~S}[\mathrm{M}+\mathrm{H}]^{+}$352.1802, found 352.1803.

9-乙酰氧基乙基-6-苠氨基-2-乙硫基嘌呤 $\left(4 b_{1}\right)$ ：白 色固体, m.p. 166 168 ${ }^{\circ} \mathrm{C}$, 产率 $85.1 \%$. ${ }^{1} \mathrm{H}$ NMR (400 $\left.\mathrm{MHz}, \mathrm{CDCl}_{3}\right) \delta 7.55(\mathrm{~s}, 1 \mathrm{H}, \mathrm{H}-8), 7.37 \sim 7.26(\mathrm{~m}, 5 \mathrm{H}$, ArH), 6.20 (bs, 1H, NH), 4.84 (bs, $2 \mathrm{H}, \mathrm{NHCH}_{2}$ ), 4.42 4.36 (m, 4H, $\left.\mathrm{NCH}_{2} \mathrm{CH}_{2} \mathrm{O}\right), 3.14$ (q, $J=7.3 \mathrm{~Hz}, 2 \mathrm{H}, \mathrm{SCH}_{2}$ ), 2.03 (s, 3H, $\left.\mathrm{COCH}_{3}\right), 1.37$ (t, $J=7.3 \mathrm{~Hz}, 3 \mathrm{H}, \mathrm{SCH}_{2} \mathrm{CH}_{3}$ ); ${ }^{13} \mathrm{C}$ NMR $\left(100 \mathrm{MHz}, \mathrm{CDCl}_{3}\right) \delta: 170.48,165.45,154.03$, $150.30,138.94,138.80,128.60,127.71,127.38,117.24$, $62.39,44.45,42.39,25.66,20.77,14.92$; IR (KBr) v: 3254, 2968, 2923, 1727, 1616, 1577, 1335, 1303, 1237, 770 $\mathrm{cm}^{-1}$; HRMS (ESI) calcd for $\mathrm{C}_{18} \mathrm{H}_{22} \mathrm{~N}_{5} \mathrm{O}_{2} \mathrm{~S}[\mathrm{M}+\mathrm{H}]^{+}$ 372.1489, found 372.1492.

9-乙酰氧基乙基-6-对氟苠氨基-2-乙硫基嘌呤 $\left(\mathbf{4} \mathbf{b}_{2}\right)$ : 白色固体, m.p. $148 \sim 150{ }^{\circ} \mathrm{C}$, 产率 $81.1 \%$. ${ }^{1} \mathrm{H}$ NMR $(400$ $\left.\mathrm{MHz} \mathrm{CDCl}_{3}\right) \delta: 7.52(\mathrm{~s}, 1 \mathrm{H}, \mathrm{H}-8), 7.33 \sim 7.27(\mathrm{~m}, 2 \mathrm{H}$, ArH), $7.01 \sim 6.95$ (m, 2H, ArH), 6.55 (bs, 1H, NH), 4.80 (bs, 2H, $\left.\mathrm{NHCH}_{2}\right), 4.42 \sim 4.34\left(\mathrm{~m}, 4 \mathrm{H}, \mathrm{NCH}_{2} \mathrm{CH}_{2} \mathrm{O}\right), 3.13$ (q, $\left.J=7.3 \mathrm{~Hz}, 2 \mathrm{H}, \mathrm{SCH}_{2}\right), 2.02\left(\mathrm{~s}, 3 \mathrm{H}, \mathrm{COCH}_{3}\right), 1.37$ (t, $\left.J=7.3 \mathrm{~Hz}, 3 \mathrm{H}, \mathrm{SCH}_{2} \mathrm{CH}_{3}\right) ;{ }^{13} \mathrm{C} \mathrm{NMR}\left(100 \mathrm{MHz}, \mathrm{CDCl}_{3}\right)$ 
$\delta: 170.46,165.49,160.89,153.93,150.36,138.98,134.59$, $129.35,129.27,117.20,115.49,115.28,62.37,43.78$, 42.41, 25.62, 20.74, 14.88; IR (KBr) v: 3255, 2924, 1726, 1616, 1579, 1333, 1302, 1241, $820 \mathrm{~cm}^{-1}$; HRMS (ESI) calcd for $\mathrm{C}_{18} \mathrm{H}_{21} \mathrm{FN}_{5} \mathrm{O}_{2} \mathrm{~S}[\mathrm{M}+\mathrm{H}]^{+}$390.1395, found 390.1397.

9-乙酰氧基乙基-6-苯乙氨基-2-乙硫基嘌呤 $\left(\mathbf{4} \mathbf{b}_{3}\right)$ : 白色固体, m.p. $117 \sim 119{ }^{\circ} \mathrm{C}$, 产率 $82.4 \%$. ${ }^{1} \mathrm{H}$ NMR (400 $\left.\mathrm{MHz}, \mathrm{CDCl}_{3}\right) \delta: 7.53(\mathrm{~s}, 1 \mathrm{H}, \mathrm{H}-8), 7.32 \sim 7.17(\mathrm{~m}, 5 \mathrm{H}$, $\mathrm{ArH}), 6.13(\mathrm{bs}, 1 \mathrm{H}, \mathrm{NH}), 4.42 \sim 4.33(\mathrm{~m}, 4 \mathrm{H}$, $\mathrm{NCH}_{2} \mathrm{CH}_{2} \mathrm{O}$ ), 3.88 (bs, 2H, $\mathrm{NHCH}_{2}$ ), 3.19 (q, $J=7.3 \mathrm{~Hz}$, $\left.2 \mathrm{H}, \mathrm{SCH}_{2}\right), 2.97$ (t, J=7.0 Hz, 2H, $\left.\mathrm{ArCH}_{2}\right), 2.03$ (s, 3H, $\left.\mathrm{COCH}_{3}\right), 1.43\left(\mathrm{t}, J=7.3 \mathrm{~Hz}, 3 \mathrm{H}, \mathrm{SCH}_{2} \mathrm{CH}_{3}\right) ;{ }^{13} \mathrm{C} \mathrm{NMR}$ $\left(100 \mathrm{MHz}, \mathrm{CDCl}_{3}\right) \delta: 170.49,165.37,154.16,139.07$, $138.78,128.82,128.61,126.45,117.30,62.42,42.37$, 41.98, 36.01, 25.65, 20.78, 15.02; IR (KBr) v: 3265, 2928, 1743, 1614, 1581, 1304, 1229, $772 \mathrm{~cm}^{-1}$; HRMS (ESI) calcd for $\mathrm{C}_{19} \mathrm{H}_{24} \mathrm{~N}_{5} \mathrm{O}_{2} \mathrm{~S}[\mathrm{M}+\mathrm{H}]^{+}$386.1645, found 386.1649 .

9-乙酰氧基乙基-6-对氟苯乙氨基-2-乙硫基嘌呤 $\left(\mathbf{4} \mathbf{b}_{4}\right)$ : 白色固体, m.p. 94 96 ${ }^{\circ} \mathrm{C}$, 产率 $85.0 \%$. ${ }^{1} \mathrm{H}$ NMR $\left(400 \mathrm{MHz}, \mathrm{CDCl}_{3}\right) \delta: 7.60(\mathrm{~s}, 1 \mathrm{H}, \mathrm{H}-8), 7.22 \sim 7.16(\mathrm{~m}$, $2 \mathrm{H}, \mathrm{ArH}), 7.01 \sim 6.96(\mathrm{~m}, 2 \mathrm{H}, \mathrm{ArH}), 5.75(\mathrm{bs}, 1 \mathrm{H}, \mathrm{NH})$, $4.43 \sim 4.35\left(\mathrm{~m}, 4 \mathrm{H}, \mathrm{NCH}_{2} \mathrm{CH}_{2} \mathrm{O}\right), 3.87$ (bs, $\left.2 \mathrm{H}, \mathrm{NHCH}_{2}\right)$, 3.17 (q, $\left.J=7.3 \mathrm{~Hz}, 2 \mathrm{H}, \mathrm{SCH}_{2}\right), 2.95(\mathrm{t}, J=7.1 \mathrm{~Hz}, 2 \mathrm{H}$, $\left.\mathrm{ArCH}_{2}\right), 2.04\left(\mathrm{~s}, 3 \mathrm{H}, \mathrm{COCH}_{3}\right), 1.42$ (t, $J=7.3 \mathrm{~Hz}, 3 \mathrm{H}$, $\left.\mathrm{SCH}_{2} \mathrm{CH}_{3}\right) ;{ }^{13} \mathrm{C}$ NMR $\left(100 \mathrm{MHz}, \mathrm{CDCl}_{3}\right) \delta: 170.50$, $165.39,162.86,160.43,154.11,138.81,134.64,130.26$, $130.18,117.25,115.51,115.30,62.41,42.39,35.20,25.63$, 20.78, 14.97; IR (KBr) v: 3269, 2926, 1740, 1613, 1581, 1327, 1302, 1226, $825 \mathrm{~cm}^{-1}$; HRMS (ESI) calcd for $\mathrm{C}_{19} \mathrm{H}_{23} \mathrm{FN}_{5} \mathrm{O}_{2} \mathrm{~S}[\mathrm{M}+\mathrm{H}]^{+}$404.1551, found 404.1554.

9-乙酰氧基乙基-6-丙氨基-2-乙硫基嘌呤 $\left(\mathbf{4} \mathbf{b}_{5}\right)$ : 白 色固体, m.p. 134 $136{ }^{\circ} \mathrm{C}$, 产率 87.5\%. ${ }^{1} \mathrm{H}$ NMR (400 $\left.\mathrm{MHz}, \mathrm{CDCl}_{3}\right) \delta: 7.60$ (s, 1H, H-8), 5.83 (bs, 1H, NH), $4.40 \sim 4.32\left(\mathrm{~m}, 4 \mathrm{H}, \mathrm{NCH}_{2} \mathrm{CH}_{2} \mathrm{O}\right), 3.57$ (bs, $\left.2 \mathrm{H}, \mathrm{NHCH}_{2}\right)$, 3.13 (q, $J=7.3 \mathrm{~Hz}, 2 \mathrm{H}, \mathrm{SCH}_{2}$ ), 2.00 (s, 3H, $\mathrm{COCH}_{3}$ ), 1.65 (sextet, $J=7.3 \mathrm{~Hz}, 2 \mathrm{H}, \mathrm{NHCH}_{2} \mathrm{CH}_{2}$ ), $1.38(\mathrm{t}, J=7.3 \mathrm{~Hz}$, $\left.3 \mathrm{H}, \mathrm{SCH}_{2} \mathrm{CH}_{3}\right), 0.96$ (t, J=7.2 Hz, 3H, $\left.\mathrm{NH}\left(\mathrm{CH}_{2}\right)_{2} \mathrm{CH}_{3}\right)$; ${ }^{13} \mathrm{C} \mathrm{NMR}\left(100 \mathrm{MHz}, \mathrm{CDCl}_{3}\right) \delta: 170.46,165.33,154.35$, 150.09, 138.63, 117.21, 62.41, 42.34, 25.60, 23.04, 20.75, 14.93, 11.43; IR (KBr) v: 3272, 2967, 2926, 1731, 1618, $1578,1302,1236 \mathrm{~cm}^{-1}$; HRMS (ESI) calcd for $\mathrm{C}_{14} \mathrm{H}_{22} \mathrm{~N}_{5^{-}}$ $\mathrm{O}_{2} \mathrm{~S}[\mathrm{M}+\mathrm{H}]^{+}$324.1489, found 324.1492.
9-乙酰氧基乙基-6-正丁氨基-2-乙硫基嘌呤 $\left(\mathbf{4} \mathbf{b}_{\mathbf{6}}\right)$ : 白色固体, m.p. $120 \sim 122{ }^{\circ} \mathrm{C}$, 产率 $82.8 \%$. ${ }^{1} \mathrm{H}$ NMR (400 $\left.\mathrm{MHz}, \mathrm{CDCl}_{3}\right) \delta: 7.59$ (s, 1H, H-8), 5.77 (bs, 1H, NH), $4.40 \sim 4.33\left(\mathrm{~m}, 4 \mathrm{H}, \mathrm{NCH}_{2} \mathrm{CH}_{2} \mathrm{O}\right), 3.60$ (bs, 2H, $\mathrm{NHCH}_{2}$ ), 3.14 (q, $J=7.3 \mathrm{~Hz}, 2 \mathrm{H}, \mathrm{SCH}_{2}$ ), 2.01 (s, 3H, $\mathrm{COCH}_{3}$ ), 1.61 (quintet, $\left.J=7.3 \mathrm{~Hz}, 2 \mathrm{H}, \mathrm{NHCH}_{2} \mathrm{CH}_{2}\right), 1.45 \sim 1.36(\mathrm{~m}, 5 \mathrm{H}$, $\left.\mathrm{NH}\left(\mathrm{CH}_{2}\right)_{2} \mathrm{CH}_{2}, \quad \mathrm{SCH}_{2} \mathrm{CH}_{3}\right), 0.92(\mathrm{t}, J=7.3 \mathrm{~Hz}, 3 \mathrm{H}$, $\left.\mathrm{NH}\left(\mathrm{CH}_{2}\right)_{3} \mathrm{CH}_{3}\right) ;{ }^{13} \mathrm{C}$ NMR $\left(100 \mathrm{MHz}, \mathrm{CDCl}_{3}\right) \delta: 170.46$, $165.34,154.32,150.07,138.62,117.23,62.41,42.34$, $40.28,31.86,25.60,20.75,20.06,14.95,13.81$; IR (KBr) $v: 3268,2956,2928,1738,1620,1579,1337,1300,1235$ $\mathrm{cm}^{-1}$; HRMS (ESI) calcd for $\mathrm{C}_{15} \mathrm{H}_{24} \mathrm{~N}_{5} \mathrm{O}_{2} \mathrm{~S}[\mathrm{M}+\mathrm{H}]^{+}$ 338.1645 , found 338.1651 .

9-乙酰氧基乙基-6-正已氨基-2-乙硫基嘌呤 $\left(\mathbf{4} \mathbf{b}_{7}\right)$ : 白色固体, m.p. $108 \sim 110{ }^{\circ} \mathrm{C}$, 产率 $80.6 \%$. ${ }^{1} \mathrm{H}$ NMR (400 $\left.\mathrm{MHz}, \mathrm{CDCl}_{3}\right) \delta: 7.60(\mathrm{~s}, 1 \mathrm{H}, \mathrm{H}-8), 5.73$ (bs, 1H, NH), $4.42 \sim 4.34\left(\mathrm{~m}, 4 \mathrm{H}, \mathrm{NCH}_{2} \mathrm{CH}_{2} \mathrm{O}\right) ; 3.60$ (bs, 2H, $\mathrm{NHCH}_{2}$ ), $3.14\left(\mathrm{q}, J=7.3 \mathrm{~Hz}, 2 \mathrm{H}, \mathrm{SCH}_{2}\right), 2.02\left(\mathrm{~s}, 3 \mathrm{H}, \mathrm{COCH}_{3}\right), 1.63$ (quintet, $\left.J=7.3 \mathrm{~Hz}, 2 \mathrm{H}, \mathrm{NHCH}_{2} \mathrm{CH}_{2}\right), 1.42 \sim 1.35(\mathrm{~m}, 5 \mathrm{H}$, $\left.\mathrm{NH}\left(\mathrm{CH}_{2}\right)_{2} \mathrm{CH}_{2}, \quad \mathrm{SCH}_{2} \mathrm{CH}_{3}\right), 1.32 \sim 1.26(\mathrm{~m}, 4 \mathrm{H}, \mathrm{NH}-$ $\left.\left(\mathrm{CH}_{2}\right)_{3} \mathrm{CH}_{2} \mathrm{CH}_{2}\right), 0.87\left(\mathrm{t}, J=6.9 \mathrm{~Hz}, 3 \mathrm{H}, \mathrm{NH}\left(\mathrm{CH}_{2}\right)_{5} \mathrm{CH}_{3}\right)$; ${ }^{13} \mathrm{C}$ NMR $\left(100 \mathrm{MHz}, \mathrm{CDCl}_{3}\right) \delta: 170.48,165.37,154.31$, $138.62,117.27,62.42,42.35,40.62,31.57,29.75,26.62$, 25.62, 22.60, 20.76, 14.96, 14.04; IR (KBr) v: 3274, 2962, 2926, 1732, 1618, 1580, 1336, 1301, $1238 \mathrm{~cm}^{-1}$; HRMS (ESI) calcd for $\mathrm{C}_{17} \mathrm{H}_{28} \mathrm{~N}_{5} \mathrm{O}_{2} \mathrm{~S}[\mathrm{M}+\mathrm{H}]^{+} 366.1958$, found 366.1972 .

\section{3 化合物 4 的抗血小板凝集活性测试}

采用健康人的血浆对 14 个 9-乙酰氧基乙基-6-烷氨 基-2-烷硫基嘌呤化合物进行了抗血小板凝集活性测试.

血浆获取方法如下: 在含有 $3.8 \%$ 柠檬酸钠的抗凝 液中加入新鲜的静脉血, 混合比例为 $6: 1(\mathrm{~V} / \mathrm{V})$, 之后 以 $300 \mathrm{r} / \mathrm{min}$ 离心 $20 \mathrm{~min}$, 得到上层富含血小板的血浆 (PRP), 下层血液再以 $900 \mathrm{r} / \mathrm{min}$ 离心 $10 \mathrm{~min}$, 得到贫血. 小板血浆(PPP).

检测方法如下: 首先在 PRP 中分别加入二甲基亚砜 和待检测化合物 (终浓度为 $100 \mu \mathrm{mol} / \mathrm{L}$ ), 在 $37{ }^{\circ} \mathrm{C}$ 下温 育 $3 \mathrm{~min}$ 后再加入二磷酸腺苷(ADP), 以 PPP 为对照放 入血小板凝集仪中测定最大血小板凝集率.其中二甲基 亚砜为阴性参照物, AR-C69931 为阳性参照物. 通过所 得到的血小板凝集率来评估待测化合物对 ADP 诱导血. 小板凝集的抵抗作用. 


\section{2 结果与讨论}

\section{1 中间体 2 和 3 及化合物 $\mathbf{4}$ 的合成}

在化合物 1 与 2 -溴乙基乙酸酯进行的亲核取代反应 中, 由于化合物 $\mathbf{1}$ 的溶解性很差, 原文献加入相转移催 化剂四丁基碘化铵来提高反应的进程与速度. 但是经实 验发现四丁基碘化铵的加入并不能显著的提高反应的 速度和产率，反而会导致后处理繁琐. 经进一步研究发 现使用碳酸钾就可以取得较好的效果, 后处理也更加容 易.

在重氮-烷硫化的反应中经 TLC 监测发现，原料 2 始终存在，即使大量使用二硫醚和延长反应时间也不能 将原料反应完全. 本文通过研究 3a 的合成来优化此反 应条件，实验结果表明，二硫醚的合适用量是 6 equiv., 反应的合适时间是 $3 \mathrm{~h}$.

实验发现, 在 3 的烷氨化反应中, 除了有目标产物 4 的生成, 还会生成 9 位酯基水解的产物. 这是因为在 三乙胺及胺化试剂的作用下, 产物 4 发生了酯的水解, 水解产物会随着时间的增长而增多. 如果延长反应时间 至 $24 \mathrm{~h}$, 便可得到水解完全的产物. 因此为了得到高产 率的目标产物就必须严格控制反应时间，尽可能的减少 水解产物的生成. 使用 TLC 监测反应进程发现, 在反应 时间为 $1.5 \mathrm{~h}$ 时, 原料化合物 3 已反应完全, 副产物仅有 少量生成.

\section{2 化合物 4 的波谱分析}

以化合物 $4 \mathbf{a}_{1}$ 为例, ${ }^{1} \mathrm{H}$ NMR 中显示 $\delta 6.72$ 处存在一 质子的宽峰归属于 $\mathrm{NH}, \delta 4.40 \sim 4.32$ 处存在四质子的多 重峰属于嘌呤环 9 位支链的 $\mathrm{CH}_{2} \mathrm{CH}_{2} \mathrm{O}, \delta 2.55$ 处存在三 质子的单峰归属于 $\mathrm{SCH}_{3}$ 以及 $\delta 2.01$ 处存在三质子的单 峰归属于乙酰基 $\mathrm{COCH}_{3}$, 在 ${ }^{13} \mathrm{C}$ NMR 中 $\delta 170.49,62.37$, $42.38,20.77$ 代表了嘌呤环 9 位支链的碳骨架, $\delta 128.58$, $127.77,127.37,44.43$ 和 14.60 分别代表了芐胺和甲硫基 碳骨架. 其 IR 谱图中, 3254 和 $1724 \mathrm{~cm}^{-1}$ 处有强峰表明 有胺基和羰基的存在. 同时 HRMS 测量值与计算值相 吻合更加印证了 $4 \mathrm{a}_{1}$ 的结构. 同理, 化合物 $\mathbf{4 a} \sim \mathbf{4 b}$ 的结 构经 ${ }^{1} \mathrm{H}$ NMR, ${ }^{13} \mathrm{C}$ NMR, IR 和 HRMS 得到确认.

\section{3 抗血小板凝集活性}

14 个化合物的抗血小板凝集活性测试结果见表 1 . 测试结果表明，在相同取代基下, $\mathbf{4 a}$ 组 $\mathbf{4 b}$ 组活性相差不 大, 活性较低. 推测其原因可能为嘌呤环 N-9 位的酯基 影响了嘌呤化合物生物活性的发挥, 文献中嘌呤化合物 的 N-9 位大多为核糖环一类的亲水性基团，所以接下来 可通过改造 N-9 位，如去除 9-位乙酰基等方式来寻求活 性更高的嘌呤化合物。
表 1 化合物 $4 \mathrm{a} \sim 4 \mathrm{~b}$ 的活性数据

Table 1 The activity data of compounds $\mathbf{4 a} \sim \mathbf{4 b}$

\begin{tabular}{|c|c|c|c|c|}
\hline Entry & Compd. & $\mathrm{R}^{1}$ & $\mathrm{R}^{2}$ & $\mathrm{PAR}_{\max }{ }^{a} / \%$ \\
\hline 1 & $4 a_{1}$ & $\mathrm{Me}$ & $\mathrm{PhCH}_{2}$ & 100.00 \\
\hline 2 & $4 a_{2}$ & $\mathrm{Me}$ & $p-\mathrm{FC}_{6} \mathrm{H}_{4} \mathrm{CH}_{2}$ & 79.23 \\
\hline 3 & $4 a_{3}$ & $\mathrm{Me}$ & $\mathrm{PhCH}_{2} \mathrm{CH}_{2}$ & 74.61 \\
\hline 4 & $4 a_{4}$ & $\mathrm{Me}$ & $p-\mathrm{FC}_{6} \mathrm{H}_{4} \mathrm{CH}_{2} \mathrm{CH}_{2}$ & 61.54 \\
\hline 5 & $4 a_{5}$ & $\mathrm{Me}$ & $n-\operatorname{Pr}$ & 60.98 \\
\hline 6 & $4 a_{6}$ & $\mathrm{Me}$ & $n$-Bu & 102.31 \\
\hline 7 & $4 a_{7}$ & $\mathrm{Me}$ & $n$-Hexyl & 103.66 \\
\hline 8 & $4 b_{1}$ & $\mathrm{Et}$ & $\mathrm{PhCH}_{2}$ & 92.68 \\
\hline 9 & $4 b_{2}$ & Et & $p-\mathrm{FC}_{6} \mathrm{H}_{4} \mathrm{CH}_{2}$ & 101.67 \\
\hline 10 & $4 b_{3}$ & Et & $\mathrm{PhCH}_{2} \mathrm{CH}_{2}$ & 93.08 \\
\hline 11 & $4 b_{4}$ & Et & $p-\mathrm{FC}_{6} \mathrm{H}_{4} \mathrm{CH}_{2} \mathrm{CH}_{2}$ & 96.15 \\
\hline 12 & $4 b_{5}$ & Et & $n-\operatorname{Pr}$ & 88.46 \\
\hline 13 & $4 b_{6}$ & Et & $n$-Bu & 77.69 \\
\hline 14 & $4 b_{7}$ & Et & $n$-Hexyl & 93.90 \\
\hline 15 & AR-C69931 & & & 0 \\
\hline
\end{tabular}

${ }^{a}$ Maximum platelet aggregation rate

\section{References}

[1] Legraverend, M. Tetrahedron 2008, 64, 8585.

[2] Matsuda, A.; Shinozaki, M.; Yamaguchi, T.; Homma, H.; Nomoto, R.; Miyasaka, T.; Watanabe, Y.; Abiru, T. J. Med. Chem. 1992, 35, 241.

[3] Sagi, G.; Otvos, L.; Ikeda, S.; Andrei, G.; Snoeck, R.; De Clercq, E. J. Med. Chem. 1994, 37, 1307.

[4] Koh, Y. H.; Shim, J. H.; Wu, J. Z.; Zhong, W. D.; Hong, Z.; Girardet, J. L. J. Med. Chem. 2005, 48, 2867.

[5] Tchilibon, S.; Joshi, B. V.; Kim, S. K.; Duong, H. T.; Gao, Z. G.; Jacobson, K. A. J. Med. Chem. 2005, 48, 1745.

[6] Laufer, S. A.; Domeyer, D. M.; Scior, T. R. F.; Albrecht, W.; Harser, D. R. J. J. Med. Chem. 2005, 48, 710.

[7] Bremond, P.; Audran, G.; Monti, H.; De Clercq, E. Synthesis 2008 3253.

[8] Elion, G. B.; Furman, P. A.; Fyfe, J. A.; Miranda, P. D.; Beauchamp, L.; Schaeffer, H. J. Proc. Natl. Acad. Sci. U. S. A. 1977, 74, 5716.

[9] De Clercq, E.; Andrei, G.; Snoeck, R.; de Bolle, L.; Naesens, L.; Degreve, B.; Balzarini, J.; Zhang, Y.; Schols, D.; Leyssen, P.; Ying, C.; Neyts, J. Nucleosides Nucleotides Nucleic Acids 2001, 20, 271.

[10] Diaz-Ricart, M.; Colar, G. Drugs Future 2008, 3, 101

[11] Springthorpe, B.; Bailey, A.; Barton, P.; Birkinshaw, T. N.; Bonnert, R. V.; Brown, R. C.; Chapman, D.; Dixon, J.; Guile, S. D.; Humphries, R. G.; Hunt, S. F.; Ince, F.; Ingall, A. H.; Kirk, I. P.; Leeson, P. D.; Leff, P.; Lewis, R. J.; Martin, B. P.; McGinnity, D. F.; Mortimore, M. P.; Paine, S. W.; Pairaudeau, G.; Patel, A.; Rigby, A. J.; Riley, R. J.; Teobald, B. J.; Tomlinson, W.; Webborn, P. J. H.; Willis, P. A. Bioorg. Med. Chem. Lett. 2007, 17, 6013.

[12] Kikugawa, K.; Iizuka, K.; Ichino, M. J. Med. Chem. 1973, 16, 358.

[13] Ingall, A. H.; Dixon, J.; Bailey, A.; Coombs, M. E.; Cox, D.; McInally, J. I.; Hunt, S. F.; Kindon, N. D.; Teobald, B. J.; Willis, P. A.; Humphries, R. G.; Leff, P.; Clegg, J. A.; Smith, J. A.; Tomlinson, W. J. Med. Chem. 1999, 42, 213.

[14] Kim, H. S.; Ohno, M.; Kim, H. O.; Choi, Y.; Ji, X. D.; Maddileti, S.; Marquez, V. E.; Harden, T. K.; Jacobson, K. A. J. Med. Chem. 2003, 46, 4974.

[15] Maerschenz, K.; Rehse, K. Arch. Pharm. Chem. Life Sci. 2006, 339, 115. 
[16] Wu, Z. J.; Li, S. L.; Ding, Z. R.; Du, H. G. Chin. J. Org. Chem. 2011, 31(10), 1618 (in Chinese).

(吴兆军, 李顺来, 丁忠仁, 杜洪光, 有机化学, 2011, 31(10), 1618.)

[17] Wang, X. Y.; Li, S. L.; Du, H. G. J. Beijing Univ. Chem. Technol.
2011, 38(6), 29 (in Chinese).

(王馨悦, 李顺来, 杜洪光, 北京化工大学学报 (自然科学版), 2011, 38(6), 29.)

[18] Wang, Z. W.; Song, S.-S.; Bao, W.; Liu, P. CN 101671249, 2010 [Chem. Abstr. 2010, 152, 453722].

(Li, L.; Lu, Z.) 